\title{
Gender Bias in Offspring Preference: Sons Still a Higher Priority, But Only in Men - Women Prefer Daughters
}

\author{
M.T. Higginson and L.W. Aarssen*
}

Department of Biology, Queen's University, Kingston, ON, K7L 3N6, Canada

\begin{abstract}
In most developed countries, gender equality and neutrality have been widely promoted and embracedthrough public policy — as a socio-cultural goal since at least the mid-twentieth century. Accordingly, we predicted that a population of highly educated youth from a relatively wealthy developed country (mostly students from a Canadian university) would display little or no significant gender bias with respect to offspring preferences. We rejected this hypothesis based on data collected in an online survey from over 2000 respondents. Participants were asked whether they had any preference regarding: (i) the gender of their firstborn child; (ii) the gender ratio of their offspring; or (iii) the gender of an only child. In all cases, there was a significant offspring gender preference, but the direction of bias depended on the respondent's gender; males significantly preferred sons whereas females significantly preferred daughters. These data show that strong gender biases in offspring preferences are still conspicuous, even within segments of modern societies where we might least expect to find them. We offer interpretations of these results in the context of evolutionary theory - as products of selection for genetic (biological) and memetic (cultural) legacy.
\end{abstract}

Keywords: Firstborn child, gender equity, memetic legacy, offspring gender preference, offspring gender ratio, only child.

\section{INTRODUCTION}

There are good reasons to expect that male and female offspring might be valued differently in humans. During the predominantly polygynous history of humans, the reproductive value of offspring is likely to have been generally higher for sons than for daughters due to their nearly limitless ability to father future offspring, as well as their potentially stronger drive to maximize fecundity because of uncertainty of paternity [1]. Male offspring may also have provided significant reproductive potential in historically patriarchal societies where males could promote offspring production through the subjugation of mates. Indeed, widespread cultural preference for sons is evident in many societies, e.g. in Bolivia [2], China [3-5], India [4, 5], Korea [4-6], Morocco [2], Pakistan [7], and in several European countries [8]. Studies have also shown preference for male inheritance of familial wealth [9] and selection for wealthy males in populations with accumulable resources [10], which may also have lead to an increased preference for sons. Recent research suggests that males may inherit the same sibling sex ratio as their fathers (i.e. a man with many brothers may have a tendency to have many sons) through a randomly segregating autosomal gene that causes segregation of male and female gametes during spermatogenesis [11].

While most previously published reports show a strong preference for sons, survey data have revealed a recent trend in females showing weaker or no preference for sons compared to male respondents [12]. A few studies have shown greater preference for female offspring in some European populations [8] - amongst pregnant women [13, 14], and

*Address correspondence to this author at the Department of Biology, Queen's University, Kingston, ON, K7L 3N6, Canada; Tel: 613-533-6133; Fax: 613-533-6617; E-mail: aarssenl@queensu.ca some undergraduate students [15]. The strength of fitness benefit associated with offspring gender may depend on environmental and cultural conditions. According to the Trivers and Willard [16] hypothesis, because resource availability is traditionally expected to have greater effects on male than on female reproductive success - especially in polygynous societies - a male in 'good condition' should outreproduce his similarly advantaged sister, while the sister should out-reproduce her brother if both are in 'poor condition' because she can marry up, while the poor brother is unlikely to win any mates at all.

Most previous research investigating offspring gender preference attributes results to socio-cultural factors $[2-5,8$, 12-15]. There are few studies addressing human reproductive choice and offspring gender preferences from an evolutionary perspective [17]. Yet, if preference for male offspring generally rewarded the reproductive success of our predecessors (because the capacity for generating grandchildren was always greater through sons than through daughters), then widespread behaviours that represent this preference can be reasonably interpreted as a product of natural selection [1]. A recent review of several studies on offspring gender selection [9] failed to find strong support for a Trivers-Willard system or any specific mechanism, but offered the hypothesis that evolution has conserved and selected for a 'module' wherein cultural preferences are reconciled with the sex ratio 'needed' (e.g. favouring males when survival depends heavily on male labour). This module may be related to the genetic mechanism proposed by Gellatly [11] and is supported by a study comparing agricultural peasants in Morocco and Bolivia [7].

Today, offspring gender preference conflicts with the ongoing mission in many nations, especially in Western Europe and North America, to pursue social and political 
agendas aimed at eliminating all discrimination on the basis of gender [18]. The cultural value or "meme" [19] of gender neutrality is especially conspicuous amongst the highly educated. Thus, unlike in previous generations, one might currently expect most young, highly-educated individuals (e.g. university students) in developed countries to have no strong preferences regarding gender issues. We explore this in the present study by testing for evidence of bias in offspring gender preference from a population surveyed at a Canadian University.

\section{METHODS}

Students, staff, faculty and alumni of Queen's University, Kingston, Ontario, Canada were invited to participate in an online survey hosted on http://www.surveymonkey.com investigating preferences surrounding offspring. Subject recruitment and data collection methods were approved by the General Research Ethics Board, Queen's University.

Respondents were asked to provide general biographic information-i.e. age, gender, marital status, cultural background, and highest level of education completed. For cultural background, respondents were instructed to choose the culture they most identified with - which may or may not have been related to their ethnic or national origins. Respondents then answered a series of questions about gender preferences in non-offspring scenarios. For example, "Would you prefer a male or female dental hygienist?" These questions were intended to allow respondents to feel accustomed to choosing one gender or another, depending on hypothetical situations. Three specific questions surrounding respondents' offspring gender preferences were asked: (i) What gender would you prefer your firstborn child to be (or did you hope for if you already have a child)? (ii) If you were to have (or do have) more than one child, would you prefer the majority to be male or female? (iii) If you were to have only one child, what gender would you prefer it to be?

Respondents were limited to the choices of "male" or "female" for all questions to eliminate the potential bias of a "no difference/no preference" option, which might be af- fected by motivations to respond according to perceived 'political correctness'. The survey instructions were clear that respondents were not obligated to respond to any questions in the survey that they found objectionable or made them feel uncomfortable. The interest, therefore was first in whether respondents were willing to answer the question (they were allowed to leave questions unanswered), and then if interested, whether they would choose "male" or "female".

After survey completion, blank survey responses (meaning a respondent accessed the survey but answered no questions) and obvious outliers (e.g. age reported as 345 years) were removed, leaving a final sample size, $n=2387$ (reduced from 2445). Data on gender preferences were analyzed using contingency chi-square $\left(\chi^{2}\right)$ to determine if respondent gender has a significant effect on offspring gender choice. Distribution $\chi^{2}$ analyses were then performed to determine the extent of the effect of respondent gender on choice. Offspring gender preference question results were also compared against the other (non-offspring) scenarios to determine if results were unique to questions about offspring. Regression analyses and median tests were performed to test for dependence on age and culture variables.

\section{RESULTS}

The online survey garnered a large number of responses (1745 females, 565 males, 77 did not identify gender). There was wide representation in respondent age (Appendix A), level of education (Appendix B) and cultural background (Appendix C), although the vast majority of respondents were Canadian undergraduate students aged 17-22 years. Sample sizes for each question varied as not all respondents answered all questions.

\section{Firstborn Child Scenario}

The choice of firstborn offspring gender was significantly dependent on the respondent's gender (Pearson's: $x^{2}=47.551$, $\mathrm{P}<0.0001, \mathrm{n}=1947$ ) (Fig. 1). Males significantly preferred sons (Pearson's: $\mathrm{x}^{2}=39.3629, \mathrm{P}<0.0001, \mathrm{n}=463$ ) and females

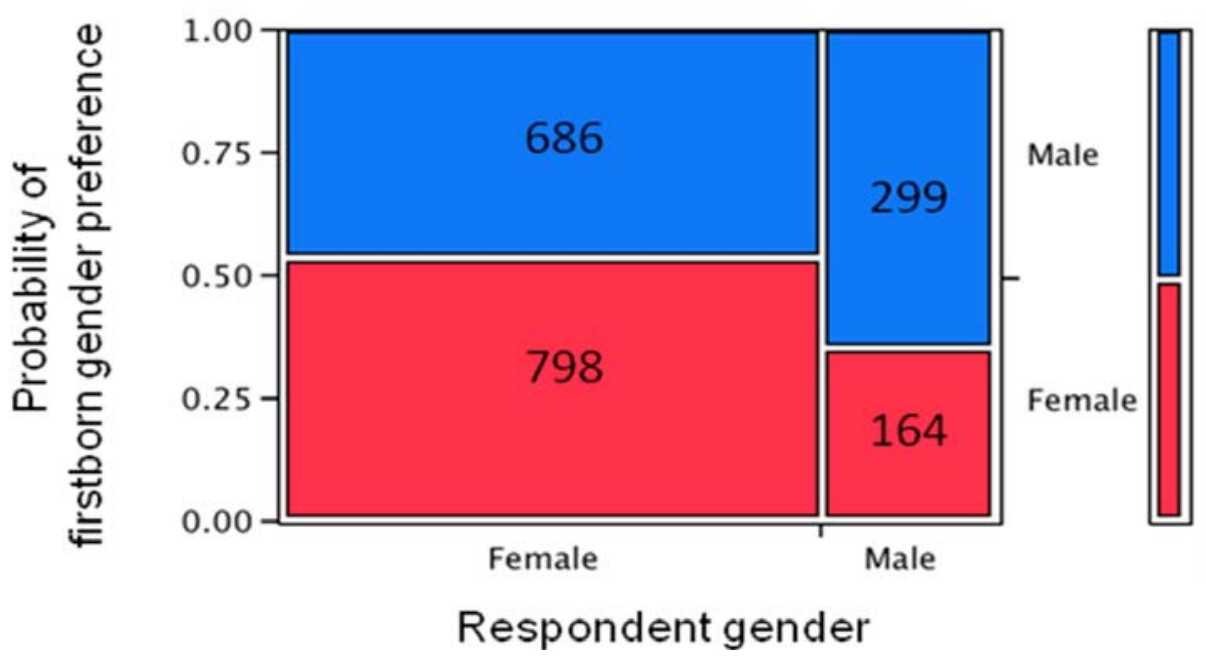

Fig. (1). Mosaic plot of probability of preferring a male versus female firstborn offspring. The X-axis shows respondent gender and the size of the division is proportional to respondent numbers (more females than males). The Y-axis on the left side shows the proportion of respondents indicating preference for male versus female firstborn offspring gender. The bar on the right side indicates overall probability and also serves as a legend for the $\mathrm{Y}$-axis. Numbers inside the plot indicate respondent counts for each group. 


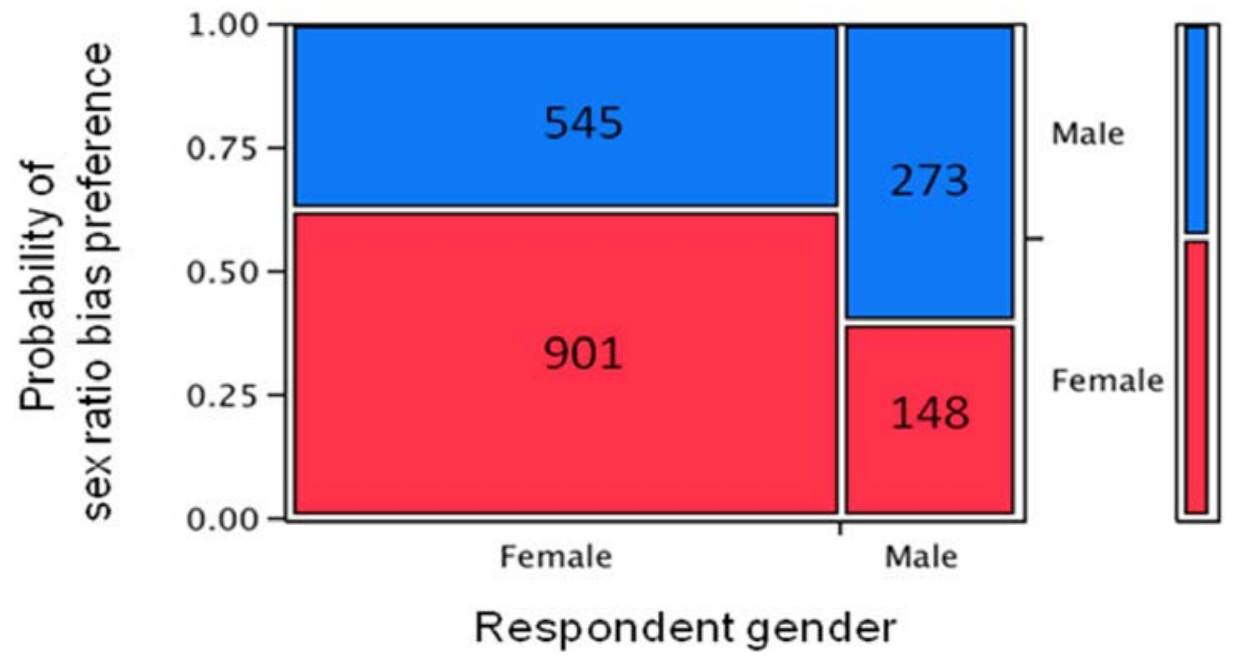

Fig. (2). Mosaic plot of probability of preferring a male- versus female-biased offspring sex ratio. The X-axis shows respondent gender and the size of the division is proportional to respondent numbers (more females than males). The Y-axis on the left side shows the proportion of respondents indicating preference for male- versus female-biased offspring sex ratio. The bar on the right side indicates overall probability and also serves as a legend for the Y-axis. Numbers inside the plot indicate respondent counts for each group.

significantly preferred daughters (Pearson's: $x^{2}=8.4528$, $\mathrm{P}=0.036, \mathrm{n}=1484)$.

\section{Offspring Sex Ratio Scenario}

The choice of a male- or female-biased offspring sex ratio was significantly dependent on the respondent's gender (Pearson's: $\mathrm{x}^{2}=73.132, \mathrm{P}<0.0001, \mathrm{n}=1897$ ) (Fig. 2). Males significantly preferred a male-biased sex ratio (Pearson's: $\left.\mathrm{x}^{2}=20.0111, \mathrm{P}<0.0001, \mathrm{n}=451\right)$ whereas females significantly preferred a female-biased sex ratio (Pearson's: $x^{2}=87.6459$, $\mathrm{P}<0.0001, \mathrm{n}=1446)$.

\section{Only Child Scenario}

The choice of gender for an only child was significantly dependent on the respondent's gender (Pearson's: $\left.\mathrm{x}^{2}=240.449, \mathrm{P}<0.0001, \mathrm{n}=1967\right)$ (Fig. 3). Males significantly preferred a son as their only child (Pearson's: $x^{2}=65.6271$, $\mathrm{P}<0.0001, \mathrm{n}=472)$ and females conversely preferred a daughter (Pearson's: $\mathrm{x}^{2}=268.0194, \mathrm{P}<0.0001, \mathrm{n}=1495$ ).

Questions about two stereotypically male occupations (firefighter and police SWAT team member) and two stereotypically female occupations (dental hygienist and nurse) were also analyzed. Both male and female respondents significantly preferred stereotypical gender roles for each of these four occupations. In two cases, male and female respondents differed significantly in their extent of preference: men more than women preferred firefighters to be male (Pearson's: $\mathrm{x}^{2}=5$.946, $\mathrm{P}=0.0148, \mathrm{n}=2049$ ), and women more than men preferred nurses to be female (Pearson's: $x^{2}=6.345$, $\mathrm{P}=0.0118, \mathrm{n}=1995)$. There was no relationship between the

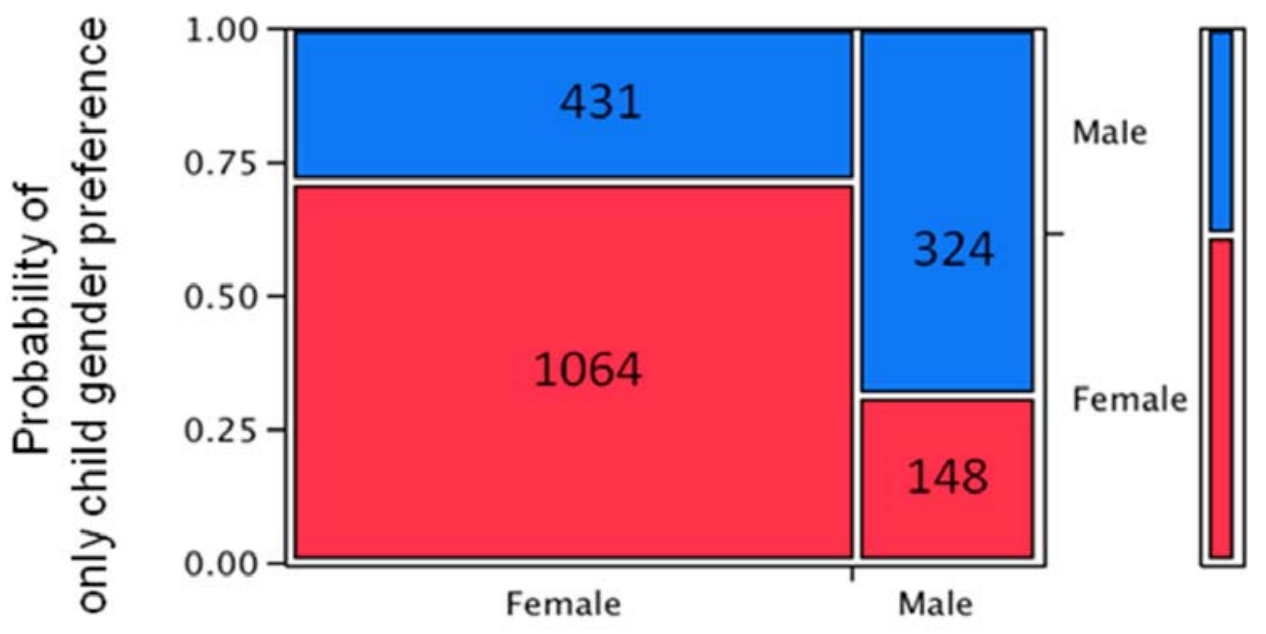

\section{Respondent gender}

Fig. (3). Mosaic plot of probability of preferring a male or female only child. The X-axis shows respondent gender and the size of the division is proportional to respondent numbers (more females than males). The Y-axis on the left side shows the proportion of respondents indicating preference for a male versus female only child. The bar on the right side indicates overall probability and also serves as a legend for the $\mathrm{Y}$-axis. Numbers inside the plot indicate respondent counts for each group. 
percentage of respondents preferring male offspring and respondent age in any scenario: firstborn child (Model I Regression: $\left.\mathrm{r}^{2}=0.03285, \mathrm{P}=0.1854, \mathrm{n}=1947\right)$, offspring sex ratio (Model I Regression: $\mathrm{r}^{2}=0.031141, \mathrm{P}=0.2018, \mathrm{n}=1897$ ), and only child (Model I Regression: $\mathrm{r}^{2}=0.042815, \mathrm{P}=0.1296$, $\mathrm{n}=1967$ ). There was also no significant difference between the percentage of respondents preferring male offspring and respondent cultural groups in any scenario (Median test: $\mathrm{P}=0.4497$ )

\section{DISCUSSION}

Gender-neutrality is now a standard cultural norm embraced within most wealthy developed countries like Canada [18]. Our results however indicate that strong gender bias in offspring preference persists even within the young highly educated population of a leading Canadian University. The large number of female respondents $(n=1745)$ relative to male respondents $(n=565)$ can be attributed to the current student population at Queen's University, which is strongly female-biased [20]. By far the largest cultural group identified was Canadian $(n=1554)$, supporting the assumption that the majority of respondents would have identified with current Canadian social values of equity and equality between the sexes. Remarkably, despite the absence of a 'no preference' option, and despite having the option to skip any uncomfortable or objectionable questions, the vast majority of respondents chose willingly to answer all questions - thus adding confidence in the honesty of answers offered. The lack of significant relationships between responses and age or cultural background is consistent with an underlying intrinsic bias in gender offspring preference.

A general preference for sons can be predicted based on the joint benefit they provide to both genetic and memetic legacies [1]. However, in all cases, offspring gender preference was significantly dependent on the respondent's gender; only males significantly preferred sons while females significantly preferred daughters (Figs. 1, 2 and 3). The preference for daughters among females is extremely strong, especially in the scenario of an only child (Fig. 3). This contrasts with the findings of some earlier research which failed to find any strong gender preference (Hank and Kohler, 2000), and augments other studies reporting a slight preference for daughters [13-15].

According to traditional theory, daughters are typically expected to confer an evolutionary fitness benefit only under poor environmental conditions [16, 21]. Given the respondent composition of the current study, however (a relatively wealthy Canadian university population), the prevailing environmental conditions may generally be considered to be 'good', with physical and economic resources in abundance.
The preference for daughters among females only therefore is particularly intriguing. Some researchers have suggested that preference for a daughter over a son may be influenced by the experience of pregnancy [14], or because of perceived mother-daughter emotional closeness, or acceptance of societal expectations and ideals for the role of women [15], including possible effects related to enhanced women's rights during the latter-half of the $20^{\text {th }}$ century [13].

These interpretations are consistent with indicators of a distinctly female 'legacy drive', which will have opportunity for display only within a population that is relatively free from patriarchal subjugation of women - as in the present study [1]. According to the 'Transmission-Competition' Hypothesis [22], when women acquire wealth and power, an evolved attraction to legacy (through meme transmission) is allowed to manifest in females as well as in males. Contemporary women in an affluent society, therefore, may have both a high predilection, as well as opportunity for satisfying legacy drive. This invites speculation that female preference for daughters may be a manifestation of a woman's desire to leave a 'close copy' of oneself - not just in terms of shared gene copies (genetic legacy), but importantly also as a medium for transmission of her uniquely feminine memetic legacy. There is currently a tendency in North American society to place a high degree of emphasis on realizing one's own 'success' through the success of one's children [23]. The present data suggest that this opportunity for legacy is envisioned for males generally through sons, but for females generally through daughters.

Our study was based on a broad, voluntary survey. Further studies may be of interest in exploring particular hypotheses for effects of age, family history, cultural identity, socio-economic status, and other demographics on offspring gender preference (and male/female parental differential) using more targeted methods, with better matched / more balanced sample size distributions for the factors of interest.

In conclusion, the gender biases in offspring preferences reported here, we suggest, represent a lingering remnant of the long history of widespread patriarchal dominance in human societies - with its evolutionary roots signaled in the present data by a persistent and conspicuous male preference for sons, and with a trend of female preference for daughters emerging as a product of the recent and rapidly spreading empowerment of women.

\section{ACKNOWLEDGMENTS}

Financial Support was provided by the National Sciences and Engineering Research Council of Canada. Christoph Richter provided helpful comments on the manuscript.

\section{Appendix A}

\begin{tabular}{|c|c|c|c|c|}
\hline Respondent's age (y) & $\mathbf{n}$ & Respondent's Age (y) & 46 \\
\hline \hline 16 & 1 & & 47 & 14 \\
\hline 17 & 107 & & 48 & 13 \\
\hline 18 & 466 & & 49 & 11 \\
\hline 19 & 376 & & 4 & 46 \\
\hline
\end{tabular}




\begin{tabular}{|c|c|c|c|}
\hline 20 & 335 & 50 & 17 \\
\hline 22 & 103 & 52 & 5 \\
\hline 23 & 38 & 53 & 11 \\
\hline 24 & 27 & 54 & 6 \\
\hline 26 & 25 & 56 & 7 \\
\hline 27 & 28 & 58 & 7 \\
\hline 28 & 23 & 59 & 5 \\
\hline 29 & 15 & 60 & 4 \\
\hline 32 & 17 & 63 & 3 \\
\hline 33 & 16 & 64 & 1 \\
\hline 34 & 17 & 65 & 4 \\
\hline 35 & 21 & 66 & 4 \\
\hline 36 & 21 & 67 & 2 \\
\hline 37 & 21 & 68 & 3 \\
\hline 38 & 18 & 69 & 1 \\
\hline 39 & 18 & 70 & 3 \\
\hline \multirow[t]{2}{*}{45} & 7 & 76 & 1 \\
\hline & & (blank) & 160 \\
\hline & & & \\
\hline
\end{tabular}

\section{Appendix B}

\begin{tabular}{|l|l|}
\hline Respondent's Highest Level of Education Achieved & $\mathbf{n}$ \\
\hline \hline Some High School & 5 \\
\hline High School Graduate & 352 \\
\hline Some College & 106 \\
\hline College Graduate & 19 \\
\hline Some Undergraduate Study & 1249 \\
\hline Bachelor's Degree & 212 \\
\hline Some Graduate Study & 55 \\
\hline Professional Degree (M.D., L.L.B./J.D. etc.) & 65 \\
\hline Master's Degree & 166 \\
\hline Ph.D. & 152 \\
\hline (blank) & 6 \\
\hline
\end{tabular}




\section{Appendix C}

\begin{tabular}{|c|c|}
\hline Respondent's Cultural Background & $\mathrm{n}$ \\
\hline Australian/New Zealander & 7 \\
\hline Canadian & 1557 \\
\hline East Asian & 195 \\
\hline European & 380 \\
\hline Latin American & 15 \\
\hline Middle Eastern & 19 \\
\hline Scandinavian & 13 \\
\hline South Asian & 63 \\
\hline South Pacific & 6 \\
\hline (blank) & 31 \\
\hline
\end{tabular}

\section{REFERENCES}

[1] Aarssen LW. Some bold evolutionary predictions for the future of mating in humans. Oikos 2007; 116: 1768-78.

[2] Crognier E, Baali A, Hilali MK, Villena M, Vargas E. Preference for sons and sex ratio in two non-western societies. American Journal of Human Biology 2006; 18: 325-34.

[3] Chan CLW, Yip PSF, Ng EHY, Ho PC, Chan CHY, Au JSK. Gender selection in China: its meanings and implications. J Rep Gen 2002; 19: 426-30.

[4] Hesketh T, Xing ZW. Abnormal sex ratios in human populations: causes and consequences. Proc Natl Acad Sci USA 2006; 103: 13271-5.

[5] Almond D, Edlund L. Son-biased sex ratios in the 2000 United States Census. Proc Natl Acad Sci USA 2008; 105: 5681-5682.

[6] Park CB. Preference for sons, family size and sex ratio: an empirical study in Korea. Demography 1983; 20: 333-52.

[7] Muhammad A. Does sex of children matter? Implications for Fertility in Pakistan. J Biosoc Sci 2009; 41: 39-50.

[8] Hank K, Kohler HP. Gender preference for children in Europe: empirical results from 17 FFS countries. Demograph Res 2000; 2: 1.

[9] Cronk L. Boy or girl: Gender preferences from a Darwinian point of view. Reprod BioMed Online 2007; 15(S2): 23-32.

[10] Nettle D, Pollet TV. Natural selection on male wealth in humans. Am Nat 2008; 172: 658-66.

[11] Gellatly C. Trends in population sex ratios may be explained by changes in the frequencies of polymorphic alleles of a sex ratio gene. Evol Biol 2009; 36: 190-200.
[12] Van Balen F. Attitudes towards sex selection in the Western world. Prenat Diagn 2006; 26: 614-8.

[13] Marleau J, Maheu M. Un garcon ou une fille? Le choix des femmes et des hommes à l'égard d'un seul enfant. Population 1998; 53: 1033-41.

[14] Marleau JD, Saucier JF. Preference for a first-born boy in western societies. J Biosoc Sci 2002; 34: 13-27.

[15] Pooler WS. Sex of child preferences among college students. Sex Roles 1991; 25: 569-76.

[16] Trivers RL, Willard DE. Natural selection of parental ability to vary the sex ratio of offspring. Science 1973; 179: 90-2.

[17] Voland E. Evolutionary Ecology of Human Reproduction. Annu Rev Anthropol 1998; 27: 347-74.

[18] Hausmann R, Tyson LD, Zahidi S. The Global Gender Gap Report. Geneva, Switzerland: World Economic Forum 2007.

[19] Dawkins R. The Selfish Gene, Rev. ed. Oxford: Oxford University Press 1989.

[20] Queen's University Office of Institutional Research and Planning. Public accountability, CUDO [Online]. 2008, [cited 2009 March 23] Available from: http://www.queensu.ca/irp/accountability/ cudo_08/SectionA.htm.

[21] Ridley M. The Red Queen: sex and the evolution of human nature. New York: Harper Collins 2003.

[22] Aarssen LW, Altman ST. Explaining below-replacement fertility and increasing childlessness in wealthy countries: legacy drive and the "transmission competition" hypothesis. Evol Psychol 2006; 4: 290-302.

[23] Marano HE. Rocking the cradle of class. Psychol Today 2005; 38(5): $52-58$. 